\title{
Laminarin Promotes Immune Responses and Reduces Lactate Dehydrogenase But Increases Glutamic Pyruvic Transaminase in Normal Mice In Vivo
}

\author{
YUNG-LUEN SHIH ${ }^{1,2,3^{*}}$, SHU-CHING HSUEH ${ }^{4,5^{*}}$, YUNG-LIANG CHEN ${ }^{6}$, \\ JIANN-SHANG CHOU ${ }^{7}$, HSUEH-YU CHUNG ${ }^{8}$, KO-LIN LIU ${ }^{1}$, HERNG-WOEI JAIR ${ }^{9}$, \\ YING-YING CHUANG ${ }^{9}$, HSU-FENG LU ${ }^{9}$, JIA-YOU LIU ${ }^{9}$ and JING-GUNG CHUNG ${ }^{10,11}$ \\ ${ }^{1}$ Department of Pathology and Laboratory Medicine, \\ Shin Kong Wu Ho-Su Memorial Hospital, Taipei, Taiwan, R.O.C.; \\ ${ }^{2}$ School of Medical Laboratory Science and Biotechnology, Taipei Medical University, Taipei, Taiwan, R.O.C.; \\ ${ }^{3}$ School of Medicine, College of Medicine, Fu-Jen Catholic University, New Taipei City, Taiwan, R.O.C.; \\ ${ }^{4}$ Division of Hematology and Oncology, ${ }^{5}$ Department of Family Medicine and Community Medicine, \\ ${ }^{7}$ Anatomic Pathology, ${ }^{9}$ Clinical Pathology, Cheng Hsin General Hospital, Taipei, Taiwan, R.O.C.; \\ ${ }^{6}$ Department of Medical Laboratory Science and Biotechnology, Yuanpei University, Hsinchu, Taiwan, R.O.C.; \\ ${ }^{8}$ Jen-Teh Junior College of Medicine, Nursing and Management, Miaoli, Taiwan, R.O.C.; \\ ${ }^{10}$ Department of Biological Science and Technology, China Medical University, Taichung, Taiwan, R.O.C.; \\ ${ }^{11}$ Department of Biotechnology, Asia University, Taichung, Taiwan, R.O.C.
}

\begin{abstract}
Background/Aim: Laminarin, a typical component of fungal cell walls, has been shown to induce immune responses in both adult and larval locusts. We investigated the effects of laminarin on immune response and glutamic oxaloacetic transaminase (GOT), glutamic pyruvic transaminase (GPT) and lactate dehydrogenase (LDH) levels in normal mice. Materials and Methods: Thirty-six normal $B A L B / c$ mice were randomly divided into four groups and treatments were provided by gavage. Group I mice acted as normal control; mice of groups II-IV received laminarin at different doses (100 $\mu \mathrm{l}$ at 1, 2.5 and $5.0 \mathrm{mg} /$ mouse in doubledistilled water, respectively). All animals were treated for 14
\end{abstract}

This article is freely accessible online.

*These Authors contributed equally to this work.

Correspondence to: Jing-Gung Chung, Ph.D., Department of Biological Science and Technology, China Medical University. No 91, Hsueh-Shih Road, Taichung 40402, Taiwan. Tel: +886 422053366 ext. 8000, Fax: +886 422053764, e-mail: jgchung@mail.cmu.edu.tw and Jia-You Liu, Department of Clinical Pathology, Cheng-Hsin General Hospital, No.45, Cheng Hsin St., Pai-Tou, Taipei 112, Taiwan, R.O.C. Tel: +886 228264400 ext. 5850, Fax: +886 228264517,e-mail: ch1835@ chgh.org.tw

Key Words: Laminarin, immune responses, glutamic pyruvic transaminase, lactate dehydrogenase. days and were weighed, blood was collected for determination of cell markers, liver and spleen samples were weighed. Spleens were used for phagocytosis and determination of natural killer (NK) cell activity and cell proliferation by flow cytometric assay. Results: Laminarin reduced the body weights and weights of liver and spleen. Laminarin increased CD3, CD19 and Mac-3 cell populations at 2.5 and $5 \mathrm{mg} / \mathrm{mouse}$, however, these did not affect CDI1b marker levels. Laminarin (1 and $5 \mathrm{mg} / \mathrm{mouse}$ ) reduced macrophage phagocytosis from peripheral blood mononuclear cells, but did not affect phagocytosis by macrophages from the peritoneal cavity. At an effector:target ratio of 50:1, laminarin reduced $N K$ cell cytotoxic activity at all levels, but at a ratio of 25:1, only at $1 \mathrm{mg}$ treatment. Laminarin did not affect T-cell and $B$-cell proliferation. Laminarin increased the level of GPT and reduced that of $\mathrm{LDH}$ at all doses, indicating laminarin can protect against liver injury. Laminarin is worthy of investigation in future experiments on improving immune responses.

It is well documented that the immune system involves sophisticated networks, and is not only responsible for protecting the host against diseases including foreign materials but also protects against toxic substances (1). Therefore, there has been focus on developing immune responses in order to protect against toxic substances entering the human body (2). In particular, many studies have paid attention to improving immune responses in humans from natural products. 
Laminarin (beta-1,3-glucan), a typical component of fungal cell walls (3) and from the algae Laminaria digitate, elicits defense-related events in tobacco and grapevine $(4,5)$ such as in partial resistance to tobacco mosaic virus or Botrytis cinerea and Plasmopara viticola, respectively $(4,6)$. In both adult and larval locusts, the injection of laminarin has been shown to induce immune responses $(7,8)$. It was reported that laminarin activates human intestinal epithelial cells to secrete proinflammatory chemokines such as interleukin-8 (IL8) and chemokine (C-C motif) ligand 2 (CCL2) in adectin-1 and spleen tyrosine kinase (SYK)-dependent manner (9).

Although several studies have reported that laminarin affects immune responses in invertebrate in vivo, to our knowledge there is no available information to show whether laminarin affects immune responses in normal mice. In the present study, we evaluated the effects of laminarin on immune responses in $\mathrm{BALB} / \mathrm{c}$ mice in vivo. We analyzed cell markers, the function of macrophages, natural killer (NK) cell activity and glutamic oxaloacetic transaminase (GOT), glutamic pyruvic transaminase (GPT), lactate dehydrogenase (LDH) levels in BALB/c mice after oral treatment with laminarin.

\section{Materials and Methods}

Materials and reagents. Laminarin and dimethyl sulfoxide (DMSO) were purchased from Sigma-Aldrich Corp. (St. Louis, MO, USA). RPMI-1640 medium, L-glutamine, penicillin-streptomycin and fetal bovine serum (FBS), were purchased from Gibco Life Technologies (Carlsbad, CA, USA). Antibodies against CD3, CD11b, CD19 and Mac-3 were purchased from BD Biosciences Pharmingen Inc. (San Diego, CA, USA). Laminarin was dissolved in double-distilled water and stored at room temperature for $1 \mathrm{~h}$ before use.

Male $B A L B / c$ mice and laminarin treatment. Thirty-six male $\mathrm{BALB} / \mathrm{c}$ mice at 4 weeks old at around 20-23 g each were purchased from the National Laboratory Animal Center (Taipei, Taiwan, ROC). Animals were kept with $12 \mathrm{~h} \mathrm{light/dark} \mathrm{cycles} \mathrm{at}$ $25^{\circ} \mathrm{C}$ in the animal center of China Medical University (Taichung, Taiwan). Before performing experiments, the animal experiments were reviewed and approved by the Institutional Animal Care and Use Committee of China Medical University (approval ID: 104-11B) and were cared for following the institutional animal ethical guidelines of the China Medical University as described previously (10). Thirty-six mice were randomly separated into four groups of nine mice. Group I mice received with $100 \mu$ l double-distilled water as control. Mice of group II-IV received with $100 \mu \mathrm{l}$ laminarin of difference dose $(1,2.5$ and $5.0 \mathrm{mg} / \mathrm{mouse}$ in double-distilled water, respectively). Laminarin was administered orally every 2 days for 14 days (total of seven times) via a steel gavage tube and the body weight was recorded. After completion of treatment, mice from each group were weighed and sacrificed as described previously (10).

Measurement of cell populations. After treatment, all mice were individually weighed and blood samples, liver and spleen were individually collected. Spleens were used for isolating splenocytes and for measuring NK cell activity as described previously (10). Blood from each mouse $(1 \mathrm{ml})$ was lysed to destroy the red blood cells with 1x Pharm Lyse ${ }^{\mathrm{TM}}$ lysing buffer (BD Biosciences Pharmingen Inc., San Diego, CA, USA) as per guidelines from BD Biosciences. Leukocytes from each mouse were collected and stained with phycoerythrin (PE)-labeled anti-mouse CD3 and CD19, fluorescein isothiocyanate (FITC)-labeled anti-mouse CD11b and Mac-3 antibodies (BD Biosciences Pharmingen Inc.) for $30 \mathrm{~min}$. All samples were washed with phosphate-buffered saline (PBS) and were analyzed by flow cytometry to measure the percentage of cell marker (population) as previously described (10).

Measurement of macrophage phagocytosis. After treatment, all macrophages were isolated from peripheral blood mononuclear cells (PBMCs) and peritoneum of each mouse from each group as described previously (11). Based on the PHAGOTEST® ${ }^{\circledR}$ kit manufacturer's instructions (Orpegen Pharma, Heidelberg, Germany), all isolated macrophages were placed in plates containing $10 \mu \mathrm{l}$ of target Escherichia coli-FITC and were individually mixed and then were analyzed for phagocytosis using flow cytometery. Quantification of phagocytosis was performed by CellQuest software as described previously (10).

Measurement of NK cell cytotoxic activity. Isolated splenocytes from each mouse were placed in 96 -well plate $\left(2.5-5 \times 10^{5}\right.$ cells/well $)$ with $100 \mu \mathrm{l}$ of RPMI 1640 medium. Target YAC- 1 cells $\left(1 \times 10^{4}\right.$ cells $)$ (Food Industry Research and Development Institute, Hsinchu, Taiwan, ROC) and PKH-67/Dil.C buffer (Sigma-Aldrich Corp.) were individually added to each well and were mixed thoroughly for $2 \mathrm{~min}$ at $25^{\circ} \mathrm{C}$. Two milliliters of PBS was added to each well for $1 \mathrm{~min}$, then $4 \mathrm{ml}$ medium was also added to the well and plates were incubated for a further $10 \mathrm{~min}$. After incubation, all samples were centrifuged at $290 \times g$ for $2 \mathrm{~min}$. NK cell cytotoxic activity was measured by flow cytometry as described elsewhere (10).

Measurement of $T$ - and B-cell proliferation. RPMI-1640 medium $(100 \mu \mathrm{l})$ was added to each well of a 96-well plate and $100 \mu \mathrm{l}$ of isolated splenocytes $\left(1 \times 10^{5}\right.$ cells/well $)$ from each mouse were added to the well. For measuring T-cell proliferation, concanavalin A (Con A, $1 \mu \mathrm{g} / \mathrm{ml}$ ) (Sigma Chemical Co., St. Louis, MO, USA) was added to the cells for stimulation for 3 days. For measuring B-cell proliferation, lipopolysaccharide (LPS, $1 \mu \mathrm{g} / \mathrm{ml}$ ) (Sigma Chemical Co.) was added to the cells for stimulation for 5 days. All samples were then measured for cell proliferation by using CellTiter 96 AQueous One Solution Cell Proliferation Assay kit (Promega, Madison, WI, USA) as previously described (10).

Measurement of blood GOT, GPT and LDH of BALB/c mice after treatment with laminarin. After treatment, blood was collected and the levels of GOT, GPT and LDH were measured using quantitative kits. LiquiUV Test (aspartate aminotransferase) for GOT, liquiUV Test (alanine aminotransferase) for GPT and liquiUV Test (lactate dehydrogenase) for LDH from Human Gesellschaft fur Biochemica und Diagnosica mbH (Wiesbaden, Germany) $(12,13)$.

Statistical analysis. Data are expressed as the mean \pm standard error (SD) of three independent experiments. For statistical comparison between laminarin-treated and control groups, differences between groups were analyzed by one-way analysis of variance and Dunnett test for multiple comparisons (SigmaPlot for Windows version 12.0; Systat Software, Inc., San Jose, CA, USA). A value of $p<0.05$ was considered to indicate a statistically significant difference. 
A

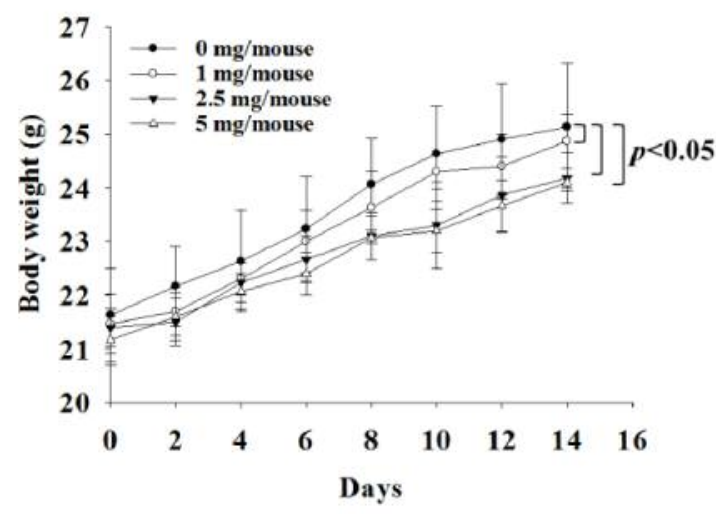

C
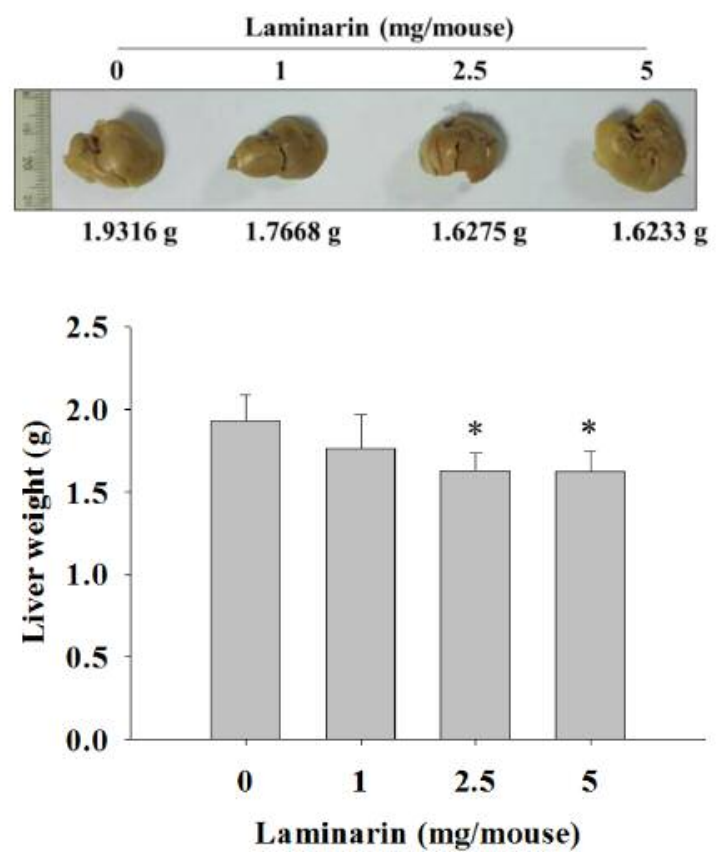

B

\begin{tabular}{lcc} 
Laminarin $(\mathrm{mg} /$ mouse $)$ \\
\hline
\end{tabular}

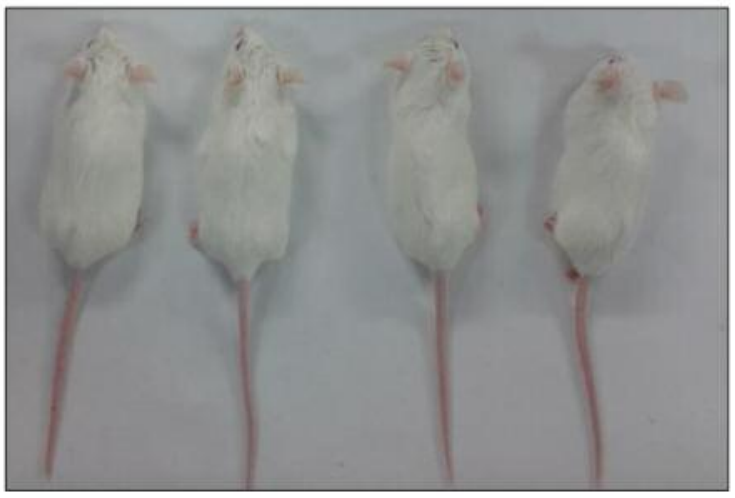

D

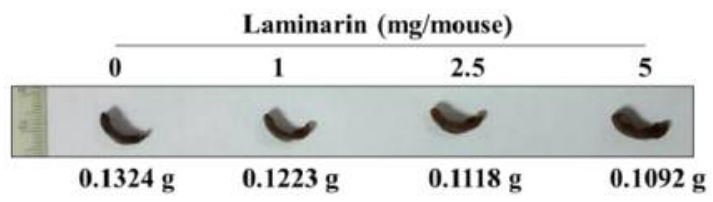

Figure 1. Effects of laminarin on body weight (A), appearance (B), liver weight $(C)$ and spleen weight $(D)$ of normal BALB/c mice. Group I: Normal control; group II: $1 \mathrm{mg} /$ mouse of laminarin; group III: $2.5 \mathrm{mg} /$ mouse of laminarin; group IV: $5 \mathrm{mg} / \mathrm{mouse}$ of laminarin. All animals were treated for 14 days. The total body weights were measured every 2 days. *Significantly different from normal control at $p<0.05$.

\section{Results}

Laminarin reduced the body weight and weights of liver and spleen from normal BALB/c mice. Representative animal appearance, body weights, and liver and spleen weights are presented in Figure 1. These results indicate that laminarin did not significantly affect animal appearance (Figure 1B) but significantly reduced body weight (Figure 1A), and liver
(Figure 1C) and spleen (Figure 1D) weights at 2.5 and 5 $\mathrm{mg} /$ mouse when compared with the control group.

Laminarin affected cell markers of white blood cells from normal $B A L B / c$ mice. Blood samples were assayed for the levels of cell markers CD3, CD19, CD11b and Mac-3 and results are shown in Figure 2. Laminarin promoted CD3 (Figure 2A) and Mac-3 (Figure 2D) expression at $5 \mathrm{mg} / \mathrm{mouse}$ 
A

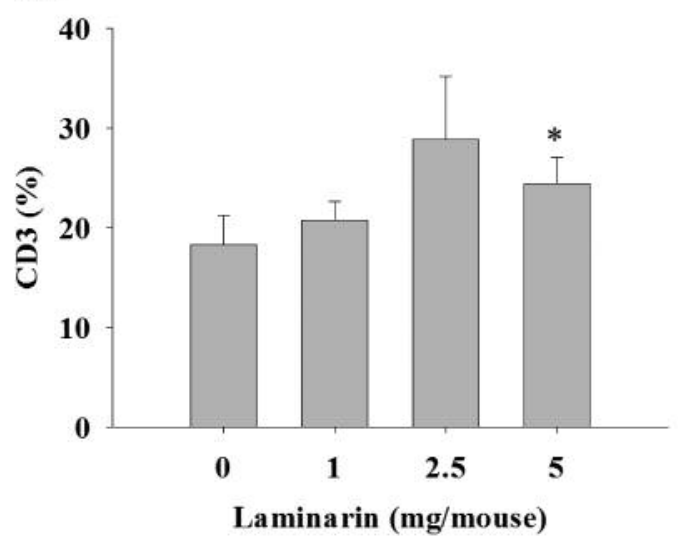

C

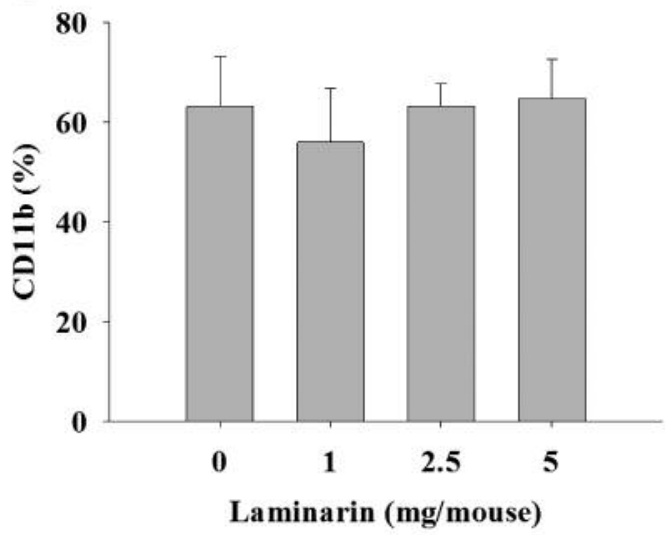

B

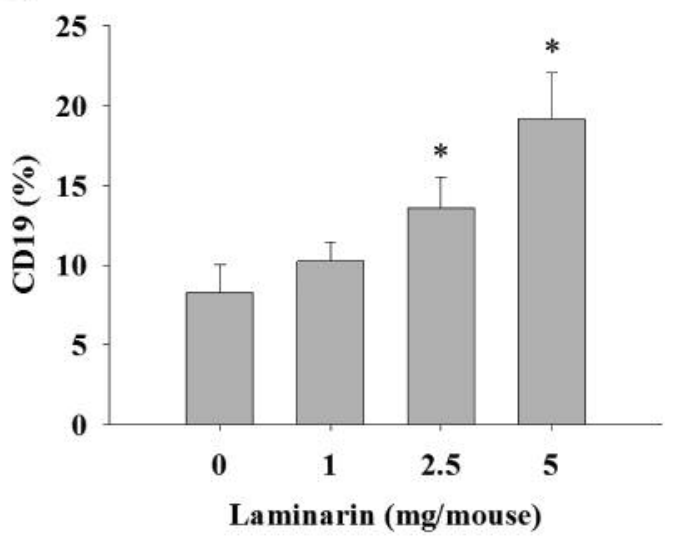

D

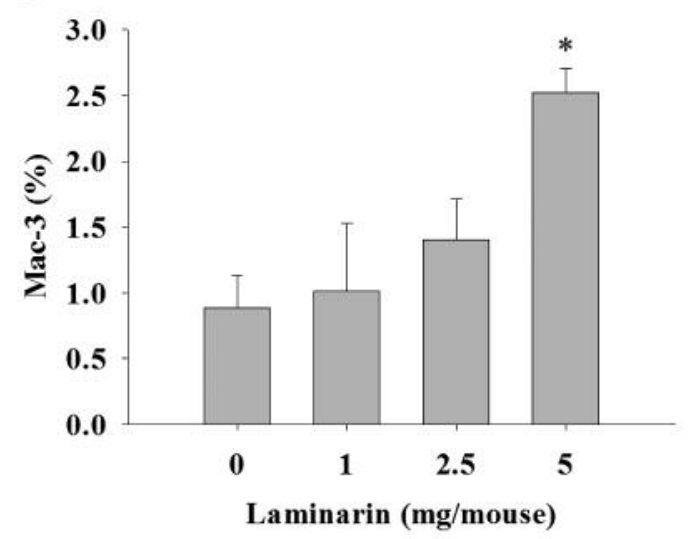

Figure 2. Effects of laminarin on cell markers in white blood cells from normal BALB/c mice. Blood was collected from all mice and were analyzed for cell markers by flow cytometry as described in the Materials and Methods. A: CD3; B: CD19; C: CD11b and D: Mac-3. *Significantly different from normal control at $p<0.05$.

A

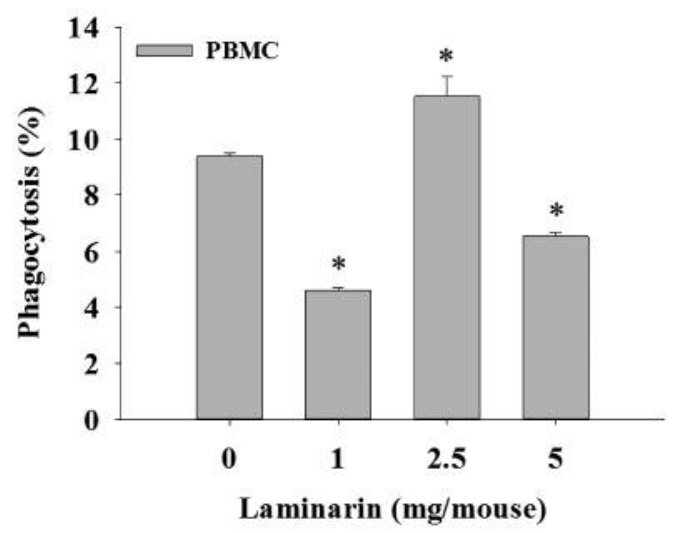

B

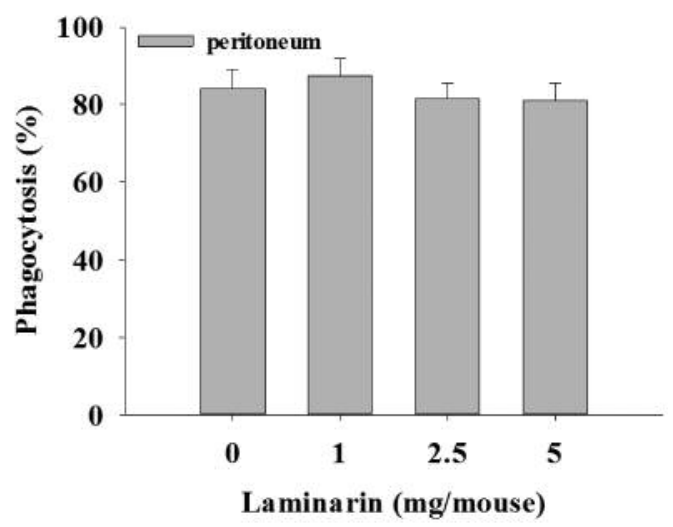

Figure 3. Effects of laminarin on macrophage phagocytosis from peripheral blood mononuclear cells (PBMCs) and peritoneal cavity of normal $B A L B / c$ mice. Blood samples were collected from mice then macrophages were isolated from PBMCs (A) and peritoneum (B) of each mouse. Phagocytosis of macrophage were measured by flow cytometry and quantified by CellQuest as described in the Materials and Methods. *Significantly different from normal control at $p<0.05$. 

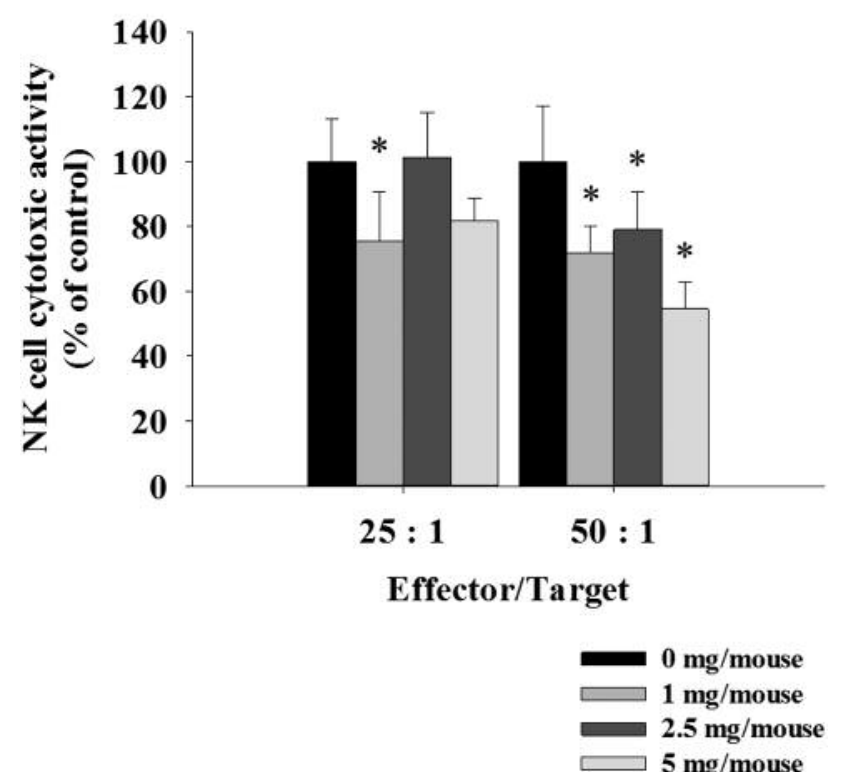

Figure 4. Effects of laminarin on cytotoxic activity of natural killer (NK) cells in normal BALB/c mice. Isolated splenocytes were placed in $1 \mathrm{ml}$ of RPMI 1640 medium in 96-well plates. Target YAC-1 cells with serumfree RPMI 1640 medium and PKH-67/Dil.C buffer was added to the cells and then NK cell cytotoxic activity was measured by flow cytometry as described in the Materials and Methods. *Significantly different from normal control at $p<0.05$.

and CD19 at 2.5 and $5 \mathrm{mg} / \mathrm{mouse}$ (Figure 2B), but did not significantly affect CD11b (Figure 2C) when compared to the control group.

Laminarin effects on phagocytosis by macrophages from $P B M C s$ and peritoneal cavity of normal BALB/c mice. After treatment, macrophages were isolated from PBMCs and the peritoneal cavity for measuring the percentage of phagocytosis. Laminarin treatment at 1 and $5 \mathrm{mg} / \mathrm{mouse}$ significantly reduced phagocytosis by macrophages from PBMCs but significantly increased at $2.5 \mathrm{mg} / \mathrm{mouse}$ treatment (Figure 3A), however, laminarin did not significantly affect phagocytosis by macrophages from the peritoneal cavity at any dose (Figure 3B).

Laminarin reduced cytotoxic activity of $N K$ cells from normal BALB/c mice. For measuring the NK cell activity, YAC-1 cells were used as targets and were assayed by flow cytometry. Results indicated that laminarin significantly reduced NK cell cytotoxic activity, at all doses at a target ratio of 50:1 but only at $1 \mathrm{mg} / \mathrm{mouse}$ at a ratio at 25:1 when compared to control treated groups (Figure 4).

Laminarin did not affect $B$ - and T-cell proliferation in normal $B A L B / c$ mice. Isolated splenocytes were assayed for B-and
A

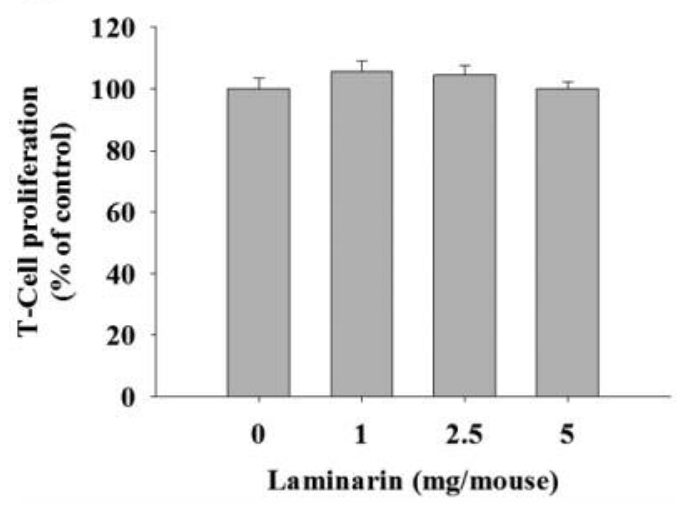

B

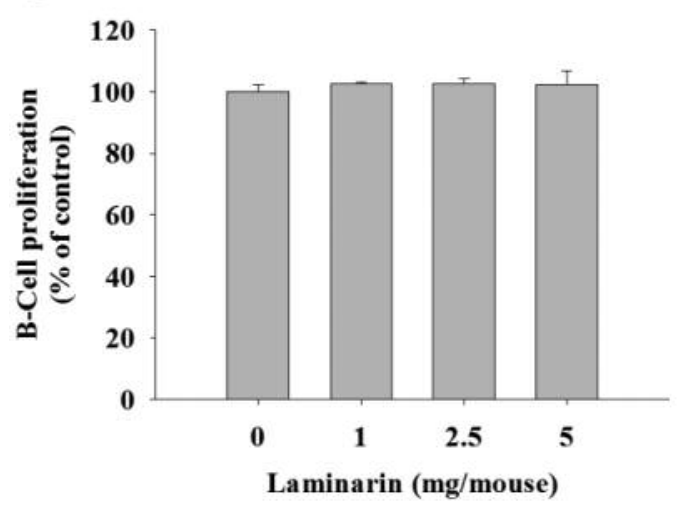

Figure 5. Effects of laminarin on B-and T-cell proliferation in normal $B A L B / c$ mice. Isolated $B$ - and $T$-cells were pretreated with concanavalin A for proliferation of T-cells (A) and with lipopolysaccharide for B-cells $(B)$, and then were analyzed by flow cytometry as described in the Materials and Methods. *Significantly different from normal control at $p<0.05$.

T-cell proliferation. Figure 5A and B indicates that laminarin did not significantly affect B- or T-cell proliferation at any dose when compared to the control group.

Laminarin effects on GOT, GPT and LDH levels of BALB/c mice. Blood sample were collected for measurement of GOT, GPT and LDH. The results indicate that laminarin increased the GOT level at $1 \mathrm{mg} / \mathrm{mouse}$ but reduced it at 2.5 and 5 $\mathrm{mg} /$ mouse (Figure 6A). All doses increased the level of GPT (Figure 6B) and reduced those of LDH (Figure 6C).

\section{Discussion}

It is interesting that an injection of laminarin can induce immune responses in locusts $(7,8)$. It was also reported that laminarin activates human intestinal epithelial cells to secrete pro-inflammatory chemokines (IL8 and CCL2) (9). Furthermore, Drosophila gram-negative bacteria binding 


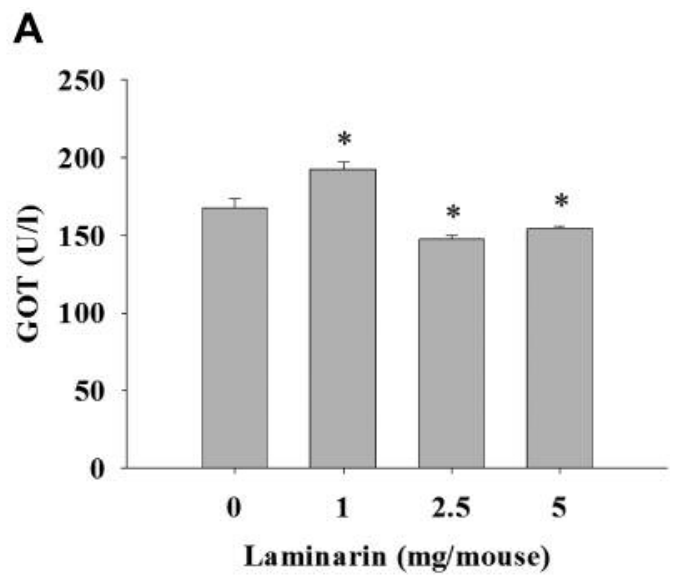

B
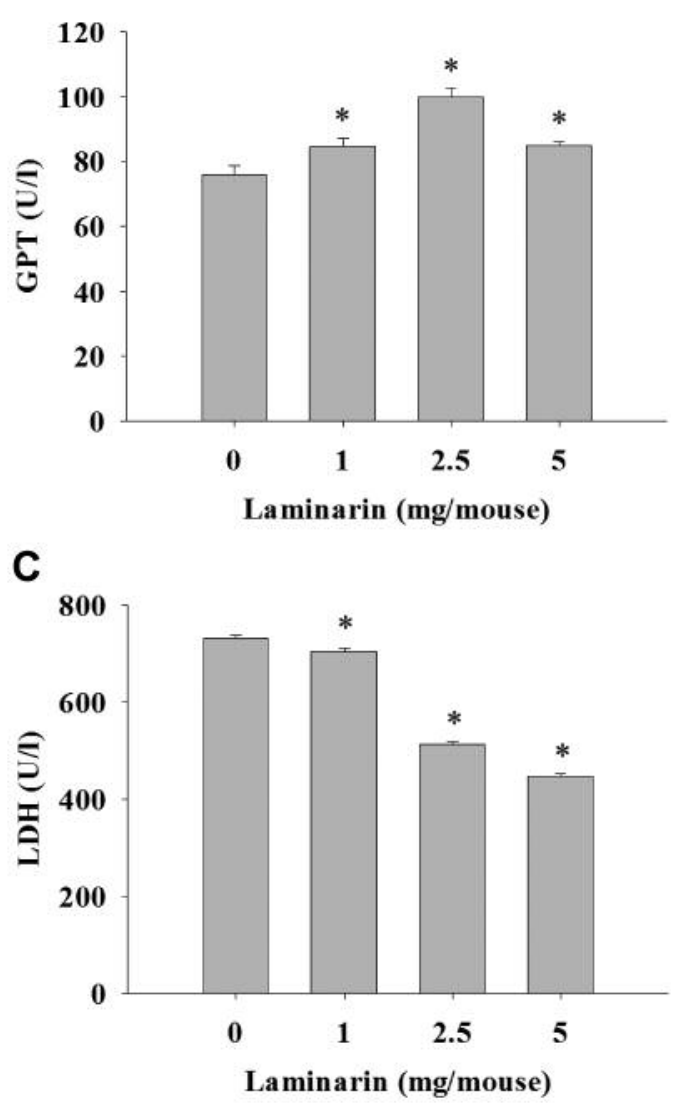

Figure 6. Effects of laminarin on serum biochemical values in normal $B A L B / c$ mice. Measurement of glutamic oxaloacetate transaminase (GOT) $(A)$, glutamic pyruvate transaminase $(G P T)(B)$ and lactate dehydrogenase $(L D H)(C)$ in the blood of $B A L B / c$ mice following exposure to laminarin. *Significantly different from normal control at $p<0.05$.

protein-1 (DGNBP-1) can bind to LPS and laminarin to enhance immune-related gene expression (11).

In particular, B- and T-cells, monocytes and macrophages play an important role for immune responses to invading foreign antigens (14). NK cells are important immune cells in innate immunity, which is related to cancer cell survival, migration and even proliferation. Results indicated that laminarin reduced NK cell cytotoxic activity at a target ratio of 50:1. Use of Yac-1 cells as a target cells for examining NK cell cytotoxic activity measurement is well documented (15).

It is well known immune responses involve multiple cell interactions such as of macrophages, B- and T-cells. Although it has been reported that laminarin induces immune responses $(7,8)$, although not in a vertebrate model, herein, we show that laminarin did not significantly affect B- and T-cell proliferation when compared to the control group of $\mathrm{BALB} / \mathrm{c}$ mice in vivo. It is well documented that concanavalin $\mathrm{A}$ is a mitogen for T-cells and LPS is for B-cells (16). Although in this study cells were pre-stimulated with these two agents, treatment with laminarin did not elicit B- or T-cell response. Apparently, further investigations are needed.

Our results indicate that laminarin did not significantly affect phagocytosis by macrophages from the peritoneal cavity (Figure 3B), while it significantly reduced that by macrophages from PBMCs (Figure 3A). Therefore, the exact effects of laminarin on Mac-3 marker associated with macrophage function need further studies.

The GPT level was increased at all doses of laminarin, while GOT was reduced at the two higher doses. Serum levels of GPT and GOT higher than normal are indicative of hepatic cell destruction (17). Thus, our finding may indicate that laminarin may protect against hepatic cell death in this mouse model. However, laminarin may also induce hepatotoxicity in mice based on increased GPT that is not clear. Further studies are needed. In patients with abdominal trauma, abnormal hepatic transaminase and LDH levels are associated with liver injury (18). Herein, we found that laminarin reduced the level of $\mathrm{LDH}$ at all doses, thus, laminarin may protect against this type of injury.

In conclusion, based on these results, we suggest that laminarin mediates immune responses through increasing B, T-, and macrophage-cell population in normal mice in vivo. However, whether or not laminarin can protect or induce hepatotoxicity still needs further investigation.

\section{Acknowledgements}

This work was supported by grant 104-40 from Cheng-Hsin General hospital, and by grant SKH-8302-105-NDR-08 from Shin Kong Wu Ho-Su Memorial Hospital, Taipei Taiwan. Experiments and data analysis were performed in part through the use of the Medical Research Core Facilities Center, Office of Research \& Development at China medical University, Taichung, Taiwan, R.O.C.

\section{References}

1 Chaplin DD: Overview of the immune response. J Allergy Clin Immunol 125: S3-23, 2010. 
2 Ermolina EV, Stadnikov AA and Smoliagin AI: Morphological features of the organs of the immune system in conditions of exposure to chromium and benzene. Gig Sanit 3: 69-71, 2012.

3 Franssens V, Simonet G, Bronckaers A, Claeys I, De Loof A and Vanden Broeck J: Eicosanoids mediate the laminarin-induced nodulation response in larvae of the flesh fly, Neobellieria bullata. Arch Insect Biochem Physiol 59: 32-41, 2005.

4 Aziz A, Poinssot B, Daire X, Adrian M, Bezier A, Lambert B, Joubert JM and Pugin A: Laminarin elicits defense responses in grapevine and induces protection against Botrytis cinerea and Plasmopara viticola. Mol Plant Microbe Interact 16: 1118-1128, 2003.

5 Klarzynski O, Plesse B, Joubert JM, Yvin JC, Kopp M, Kloareg B and Fritig B: Linear beta-1,3 glucans are elicitors of defense responses in tobacco. Plant Physiol 124: 1027-1038, 2000.

6 Menard R, Alban S, de Ruffray P, Jamois F, Franz G, Fritig B, Yvin JC and Kauffmann S: Beta-1,3 glucan sulfate, but not beta1,3 glucan, induces the salicylic acid signaling pathway in tobacco and Arabidopsis. Plant Cell 16: 3020-3032, 2004.

7 Goldsworthy G, Opoku-Ware K and Mullen L: Adipokinetic hormone enhances laminarin and bacterial lipopolysaccharideinduced activation of the prophenoloxidase cascade in the African migratory locust, Locusta migratoria. J Insect Physiol 48: 601-608, 2002.

8 Mullen LM and Goldsworthy GJ: Immune responses of locusts to challenge with the pathogenic fungus Metarhizium or high doses of laminarin. J Insect Physiol 52: 389-398, 2006.

9 Cohen-Kedar S, Baram L, Elad H, Brazowski E, Guzner-Gur H and Dotan I: Human intestinal epithelial cells respond to beta-glucans via Dectin-1 and Syk. Eur J Immunol 44: 3729-3740, 2014.

10 Lu HF, Tung WL, Yang JS, Huang FM, Lee CS, Huang YP, Liao WY, Chen YL and Chung JG: In vitro suppression of growth of murine WEHI-3 leukemia cells and in vivo promotion of phagocytosis in a leukemia mice model by indole-3-carbinol. $\mathrm{J}$ Agric Food Chem 60: 7634-7643, 2012.

11 Kim YS, Ryu JH, Han SJ, Choi KH, Nam KB, Jang IH, Lemaitre B, Brey PT and Lee WJ: Gram-negative bacteria-binding protein, a pattern recognition receptor for lipopolysaccharide and beta1,3-glucan that mediates the signaling for the induction of innate immune genes in Drosophila melanogaster cells. J Biol Chem 275: 32721-32727, 2000 .
12 Recommendations of the German Society for Clinical Chemistry. Standardization of methods for the determination of enzyme activities in biological fluids. Z Klin Chem Klin Biochem 8: 658-660, 1970

13 Nagamatsu Y, Yamamoto J, Fukuda A, Ohta M, Tsuda Y and Okada Y: Determination of leukocyte elastase concentration in plasma and serum by a simple method using a specific synthetic substrate. Haemostasis 21: 338-345, 1991.

14 Arpinati $\mathrm{M}$ and Curti A: Immunotherapy in acute myeloid leukemia. Immunotherapy 6: 95-106, 2014.

15 Fan MJ, Yeh PH, Lin JP, Huang AC, Lien JC, Lin HY and Chung JG: Anthocyanins from black rice (Oryza sativa) promote immune responses in leukemia through enhancing phagocytosis of macrophages in vivo. Exp Ther Med 14: 59-64, 2017.

16 Yeh MY, Shih YL, Chung HY, Chou J, Lu HF, Liu CH, Liu JY, Huang WW, Peng SF, Wu LY and Chung JG: Chitosan promotes immune responses, ameliorates glutamic oxaloacetic transaminase and glutamic pyruvic transaminase, but enhances lactate dehydrogenase levels in normal mice in vivo. Exp Ther Med 11: 1300-1306, 2016.

17 Yamashita T, Ohshima H, Asanuma T, Inukai N, Miyoshi I, Kasai N, Kon Y, Watanabe T, Sato F and Kuwabara M: The effects of alpha-phenyl-tert-butyl nitrone (PBN) on copperinduced rat fulminant hepatitis with jaundice. Free Radic Biol Med 21: 755-761, 1996

18 Bilgic I, Gelecek S, Akgun AE and Ozmen MM: Predictive value of liver transaminases levels in abdominal trauma. Am $\mathbf{J}$ Emerg Med 32: 705-708, 2014.

Received February 2, 2018

Revised February 27, 2018

Accepted March 6, 2018 